\title{
POINT-AXIAL MASS DISTRIBUTION IN THE EXTERNAL GALACTIC BULGE
}

\author{
J. SANZ-SUBIRANA ${ }^{(1)}$ and J.M. JUAN-ZORNOZA ${ }^{(2)}$
}

(1) Dept. de Matemàtica Aplicada i Telemàtica. ${ }^{(2)}$ Dept. de Física Aplicada Universitat Politècnica de Catalunya. Apdo. 30.002 - Barcelona. SPAIN

EAN: MATJSS@MAT.UPC.ES

\section{Introduction}

The main purpose in this work is to present some analytical results about the spatial distribution of the stars in the Galactic Bulge. These results have been obtained by considering that the stellar system verifies the collisionless Boltzman equation and the ellipsoidal hypothesis (non-axisymmetrical) for the distribution of peculiar velocities of the stars.

A qualitative study of the kinematical behavior and mass distribution for an axisymmetric multi-component galactic model is presented "by Cubarsi \& Hernández-Pajares in this Symposium [1]. The angular dependence for a stellar system characteristic of the external galactic bulge is studied in our work by considering a point-axial velocity distribution function.

The general solution for the potential in a stationary point-axial system model can be found in [2], also, the separable potentials in the time-depending models can be found in [3]. These potential functions are compatible with triaxial spatial distribution of stars when the hydrodymamical equations are fulfilled.

\section{The galactic model}

We adopt the galactic model based on the collisionless Boltzman equation:

$$
\frac{\mathrm{d} \Psi}{\mathrm{d} \mathrm{t}}=\frac{\partial \Psi}{\partial \mathrm{t}}+\underline{\mathrm{V}} \cdot \nabla_{\underline{\underline{I}}}^{\Psi}-\nabla_{\underline{\underline{ }}} \mathbf{U} \cdot \nabla_{\underline{\underline{v}}} \Psi=0
$$

and an ellipsoidal hypothesis for the distribution of peculiar velocities of stars:

$$
\Psi(\underline{\mathrm{r}}, \underline{\mathrm{v}}, \mathrm{t}) \equiv \Psi(\mathrm{Q}+\sigma)=\mathrm{e}^{-1 / 2}(\mathrm{Q}+\sigma)
$$

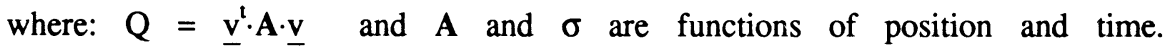

Equation (1) under hypothesis (2) gives the well known Chadrasekhar equations [4], which leads to the elements of the velocity ellipsoid. A more precise description of the model is presented in [5]. The model admits the following potential function separable in $\omega$ and $Z$ :

$$
\mathbf{U}=\frac{\mathrm{D}_{1}}{2 \mathrm{k}_{3}^{2}}\left(\omega^{2}+\mathrm{Z}^{2}\right)+\mathrm{ctt} \text {. }
$$

The scalar function $\sigma$ is invariant along of the local centroid trajectories [5], which is related to the mass distribution by :

$$
v=\iiint_{v} \Psi(Q+\sigma) d v=\frac{2 \pi}{|A|^{1 / 2}} e^{-\sigma / 2}
$$


The determinant $|\mathbf{A}|$ is a fourth degree polinomy in $\omega$ and $z$, which coefficients are functions of $2 \theta$, and

$$
\sigma=\frac{D_{1}}{k_{3}^{2}}\left(a(2 \theta) \omega^{2}+k_{3} z^{2}\right)-\frac{\gamma^{2}}{k_{2}} \frac{a(2 \theta) \omega^{2}}{\left(1+p^{2}\right) \kappa^{2}+a(2 \theta) \omega^{2}}
$$

where

$$
K^{2}=\left(K_{1}^{2}-Q^{2}\right) / k_{2} \quad ; \quad a(2 \theta)=K_{1}+Q \sin (2 \theta+\phi),
$$

being $D_{1}, k_{2}, \gamma$ constants, and the others parameters are arbitrary functions of time.

Taking into account (5), the stellar density (4) in the Galactic Plane can be written as:

$$
v=\frac{\exp \left(-\frac{D_{1}}{2 k_{3}^{2}} a(2 \theta) \omega^{2}+\frac{\gamma^{2}}{2 k_{2}} \frac{a(2 \theta) \omega^{2}}{\kappa^{2}+a(2 \theta) \omega^{2}}\right)}{\left(k_{2}\left(k^{2}+a(2 \theta) \omega^{2}\right)\left(k_{3}+p a(2 \theta) \omega^{2}\right)\right)^{1 / 2}}
$$

\section{3.- Discussion}

1) The stellar density (7) is characterized by two differentiated contributions in the exponential factor, both functions of the azimuthal angle $\theta$ :

- The first term is due for the harmonic potential and takes into account the anisotropic distribution of velocities. Being $D_{1}$ positive, this term fixes the scale length of the bulge.

- The second term is associated to the rotation of the stellar system. It produces a displacement forward of the mass density maximum. If the system is fast rotating, it can predominate in front of the other.

2) For a non-rotating system $(\gamma=0)$, the isodensity surfaces are ellipsoids which axis-ratios are determined from the parameters of the velocity distribution. Nevertheless, in a rotating system the isodensity surfaces shows bulk shapes and also elliptic-toroidal shapes. The isodensity curves in a fixed meridian section are Cassini ovals.

For the numerical application, we have taken the values that corresponds to the last estimations publishes in the literature $[6,7,8,9]$.

\section{BIBLIOGRAPHY}

[1] Cubarsí et al.: 1992, This Symposium.

[2] J.M. Juan-Zornoza et al.:1991, Astrophys. Space Sci. 185, 95

[3] J. Sanz et al.:1987, Proc. IAU Twenty European Regional Astronomy Meeting 4, 227.

[4] Chandrasekhar, S.: 1942, Principles of Stellar Dynamics, The University of Chicago Press, Chicago.

[5] Sanz et al.: 1989, Astrophys. and Space Sci. 156, 19.

[6] T.D. Kinman et al.: 1989, Astroph. J. 95, 804.

[7] R.M. Rich: 1992, Proc. IAU Symposium n¹49, 29.

[8] R. Sharples et al.: 1990, M.N.R.S.A. 246, 54.

[9] S. Ninkovich: 1987, Astrophys. and Space Sci. 136, 299. 